\title{
Ist der Verbwortschatz entscheidend für die Makrostruktur? Eine Analyse anhand elizitierter Narrative deutsch-russisch bilingualer Vor- und Grundschulkinder
}

Is the verb lexicon decisive for the macrostructure? An analysis based on elicited narratives of German-Russian bilingual preschoolers and school children

\author{
Natalie Sürmeli \\ Humboldt-Universität zu Berlin / Universität Potsdam, Deutschland
}

This study explores the relation between the development of narrative skills at the macrostructural level and the productive lexical abilities (verbs) of German-Russian children. The narratives are elicited using the Multilingual Assessment Instrument for Narratives (MAIN) and the lexical abilities are assessed using different tests. Twenty-one preschoolers (mean age: $3 ; 9$ ), forty-four 1st graders (mean age: 6;11) and twenty-two 3rd graders (mean age: 9;3) were included in the study. Correlation analyses were performed between verb lexicon and the following macrostructural components: Story Structure, Structural Complexity and Internal State Terms. The analysis also targets cross-language effects. In addition, the production of verbs within the elicited narratives was taken into account. Some positive correlations were found; however, no clear pattern across age groups and languages was observed. It is suggested that cognitive abilities might be a more decisive factor than lexical abilities and/or that the verbs assessed via the vocabulary tests are more specific than the ones required to achieve high scores for macrostructure.

\section{$1 \quad$ Einführung}

Es gibt eine Reihe besonderer Merkmale, die in ihrer Gesamtheit die menschliche Sprache von Kommunikationssystemen anderer Lebewesen unterscheiden (Hocket, 1960). Zu diesen Charakteristika gehört die Möglichkeit, sich auf vergangene Zeiten und ferne Orte zu beziehen. Tatsächliche, mögliche und irreale Ereignisse können sprachlich ausgedrückt werden. Der Mensch kann fiktionale Wesen und Welten erfinden, philosophische Gedankenexperimente durchführen 
und er kann berichten, was sich gestern oder vor 800 Jahren ereignet hat. Er kann Geschichten erzählen.

Diese Fähigkeit ist sogar von elementarer Bedeutung, denn narrativer Diskurs ist eine allgegenwärtige Form der menschlichen Kommunikation und die Basis für das Funktionieren in der Gesellschaft (Bruner, 1986; McCabe, 1996). Sprache, insbesondere Diskurs, ist das Hauptmedium, durch das akademisches Wissen überliefert und erworben wird (Roth, 1986). Als Brücke zwischen mündlicher und schriftlicher Sprache stellt narrativer Diskurs eine wichtige Basis für den akademischen Erfolg und besonders für das Erlernen von Lese- und Schreibfähigkeiten dar (Westby, 1991; Swanson et al., 2005). Zahlreiche Studien belegen, dass die Fähigkeit, eine kohärente Geschichte zu erzählen, essenziell für den schulischen Erfolg ist (Bishop \& Edmundson, 1987; Hayward \& Schneider, 2000). Außerdem erfüllt Erzählen auch wichtige Funktionen wie zum Beispiel affektive Entlastung (Quasthoff, 1980). Bei einem so komplexen Prozess wie der Entwicklung narrativer Fähigkeiten sind viele unterschiedliche Einflussfaktoren denkbar: beispielsweise ob einem Kind viel oder wenig vorgelesen wird, ob es von seinen Bezugspersonen zum Erzählen ermuntert wird, die lexikalischen und grammatischen Fähigkeiten und die kognitive Entwicklung des Kindes.

Ziel der vorliegenden Studie ist es, zu untersuchen, inwiefern es einen Zusammenhang zwischen der Entwicklung der narrativen Fähigkeiten auf makrostruktureller Ebene und dem Verberwerb gibt. Der Verberwerb wird hierbei auf semantisch-lexikalischer Ebene betrachtet. Der Fokus auf Verben ist vor allem darin begründet, dass Handlungen und Ereignisse primär durch Verben ausgedrückt werden - und eben diese sprachlich kodieren zu können, ist eine Voraussetzung, um Geschichten erzählen zu können.

Im Folgenden werden zunächst narrative Fähigkeiten und deren Erwerb behandelt. Danach wird auf die Motivation, den Verbwortschatz im Zusammenhang mit der Makrostruktur zu untersuchen, näher eingegangen. Ergänzend wird ein kurzer Überblick über den Erwerb von Verben gegeben. Darauf basierend werden anschließend Fragestellungen und Hypothesen formuliert, die anhand von deutsch-russisch bilingualen Kindern verschiedener Altersgruppen empirisch untersucht werden sollen. Die Bilingualität der Probanden ermöglicht einen Vergleich zwischen ihren beiden Sprachen. 


\section{Narrative Fähigkeiten}

\subsection{Begriffsklärung}

Im alltäglichen und umgangssprachigen Gebrauch ist die Bedeutung des Begriffs Erzählung ${ }^{1}$ recht breit gefasst. In der Forschung wird sie je nach zugrundeliegender Theorie oder gesetztem Fokus entsprechend eindeutiger definiert. Als Basis für diese Studie wird die folgende Begriffsbestimmung herangezogen: „Eine Erzählung oder Geschichte ist eine systematische Strukturierung von thematisch orientierten Einzelheiten, die erfunden oder erlebt sein können" (Schelten-Cornish, 2008:9). Es wird zudem betont, dass eine Erzählung mehr als eine Beschreibung ist: Eine Beschreibung könne als Vorstufe oder auch Teil der Geschichte betrachtet werden. Als entscheidendes Kriterium für eine Geschichte nennt Schelten-Cornish (2008) die Weiterentwicklung.

\subsection{Mikro- und Makrostruktur}

Grundsätzlich differenziert die Erzählforschung zwischen zwei Ebenen: Mikround Makrostruktur. Diese können getrennt voneinander analysiert werden, aber auch hinsichtlich ihrer wechselseitigen Beziehung (Liles, Duffy, Merritt, \& Purcell, 1995).

Die Mikrostruktur konzentriert sich auf sprachliche Mittel zur strukturellen Organisation des Textes bzw. der Erzählung. Analysen auf mikrostruktureller Ebene untersuchen unter anderem morphosyntaktische Aspekte und Kohäsionsmittel wie zum Beispiel Konnektive, Tempus, Situationsdeixis und Substitutionen durch Pro-Formen. Die Mikrostruktur ist sprachspezifisch.

Die Makrostruktur stellt die globale Struktur der Geschichte dar (Heilmann, Miller, \& Nockerts, 2010; Mandler, 1979) und ist weniger sprachabhängig. Stattdessen kommt hier die kognitive Komponente stärker zum Tragen (Liles, 1993).

Diese Studie befasst sich mit den drei verschiedenen Komponenten der Makrostruktur, wie sie im Multilingual Assessment Instrument for Narratives (MAIN) beschrieben sind: Story Structure (SS), Structural Complexity (SC) und Internal State Terms (IST) (Gagarina et al., 2012 und 2015). ${ }^{2}$ SS ist ein quantitatives Maß, das (u.a.) basierend auf dem Story Grammar Modell (Stein \& Glenn, 1979) die Teile einer Geschichte berücksichtigt. Eine Geschichte ist aufgeteilt in Episoden, die jeweils wiederum aus verschiedenen Elementen

1 Die Begriffe Erzählung und Geschichte werden in diesem Artikel synonym verwendet.

2 MAIN bietet Bildergeschichten, die unter Berücksichtigung vieler Faktoren eigens zur Elizitierung und Auswertung fiktionaler Narrativen entwickelt wurden. 
bestehen. Diese Teile hängen miteinander zusammen bzw. bauen aufeinander auf. Sie können aber unabhängig voneinander und auch in unterschiedlicher Reihenfolge realisiert werden. Zunächst ist zu klären, was mit den Teilen einer Geschichte gemeint ist. Angelehnt an Stein \& Glenn (1979) nennt ScheltonCornish als Teile einer Geschichte (Schelton-Cornish, 2008:12):

1. Kulisse

2. Verursachendes Geschehen

3. Plan

4. Lösungsversuch, Aktion

5. Interne Reaktion (kann fast an jeder Stelle vorkommen)

6. Ergebnis der Aktion

7. Zusammenfassung; Gedanken und Gefühle; Protagonist hat gelernt; Reflexion über Zukunft; Moral

Die Teile 2 bis 6 ordnet Schelton-Cornish dabei dem Hauptteil der Geschichte zu. Die Mehrheit dieser Aspekte findet sich auch in den Bildergeschichten bei MAIN wieder. Die folgende Auflistung zeigt die Parallelen zu den Elementen der SS:

1. Kulisse: entspricht bei MAIN dem Setting.

3. Plan: ist vergleichbar mit Goal

4. Lösungsversuch, Aktion: entspricht in MAIN Attempt

5. Interne Reaktion: Dieser Aspekt ist bei der SS untergliedert in IST as initiating event - das heißt ein innerer Zustand, der ein Ereignis initiiert - und IST as reaction, ein innerer Zustand als Reaktion, der aus einem Ereignis resultiert.

6. Ergebnis der Aktion: wird bei MAIN als Outcome bezeichnet.

Die $S S$ einer Geschichte setzt sich also zusammen aus Episoden, die jeweils IST as initiating event, Goal, Attempt, Outcome und IST as reaction enthalten können. Die Bildergeschichten bei MAIN umfassen jeweils drei solcher Episoden. Zusätzlich fließt das Setting, das heißt die räumliche und zeitliche Einordnung der Geschichte, mit ein.

Eine weitere Komponente der Makrostruktur stellt die $S C$ dar. Sie ist ein qualitatives $\mathrm{Ma}$ der Struktur der Geschichte, welches eng mit der SS zusammenhängt: Sie ergibt sich aus neun der 17 Elemente, welche die $S S$ bei MAIN ausmachen. Für die SC werden nur Goals, Attempts und Outcomes betrachtet. Wird für jede der drei Episoden beispielsweise Attempt, Outcome oder beides produziert, so zeigt dies zwar quantitativ gesehen eine entwickelte Makrostruktur, jedoch keine ausgeprägte Komplexität der Geschichte. Eine vollständige Episode zeichnet aus, dass sie sowohl Goal, Attempt als auch 
Outcome enthält (Gagarina et al., 2012, referierend auf den Binary Decision Tree von Westby, 2005). Eine solche komplette Episode wird im Folgenden abgekürzt als $G A O$ bezeichnet. IST bilden die dritte der hier untersuchten Komponenten. $\mathrm{Zu}$ ihnen gehören Ausdrücke der Wahrnehmung (Perceptual state terms), Beschreibungen physiologischer Zustände (Physiological state terms), Ausdrücke des Bewusstseins (Consciousness terms), Emotionsausdrücke (Emotion terms) sowie Verben wie wissen, wollen oder hoffen und sogenannte linguistische Verben, wie zum Beispiel rufen (Gagarina et al., 2012).

\subsection{Erwerb narrativer Fähigkeiten}

Die Entwicklung von narrativen Fähigkeiten ist ein komplexer Prozess, der Zeit und qualitativ hochwertigen Input voraussetzt. Neben sprachlichem Wissen sind dazu noch andere Kompetenzen erforderlich: ein dem Alter entsprechend entwickeltes Weltwissen und die Fähigkeit, kausale Zusammenhänge zu erkennen und logische Schlussfolgerungen zu ziehen (Schelton-Cornish, 2008:13). Von zentraler Bedeutung ist auch die Fähigkeit zur Theory of Mind. Das beinhaltet zum Beispiel, einschätzen zu können, welchen Wissensstand der Zuhörer hat (Schelton-Cornish, 2008:13).

$\mathrm{Ab}$ dem Alter von 30 Monaten kann eine general event representation festgestellt werden (Hudson \& Shapiro, 1991). Durch die Erfahrungen, die das Kind macht, entwickelt es mentale Schemata, die Ereignisse repräsentieren. Mit etwa 4 bis 5 Jahren können Handlungs- und Ereignisabfolgen verbalisiert werden. Das ist vor allem der Fall, wenn Kindern dazu entsprechende Bilder gezeigt werden (Hughes et al., 1997). Etwa ab dem Schulalter können Kinder Geschichten mit sechs Teilen, wie sie oben beschrieben sind, erzählen; es müssen jedoch nicht alle ihre Erzählungen so viele Teile enthalten (Schelton-Cornish, 2008:16). Geschichten, die Emotionen und Gedanken der Charaktere beinhalten und vollständige Episoden aufweisen, sind von etwa 8-jährigen Kindern erwartbar (Hughes et al., 1997; Liles 1987). Die Produktion erster IST beginnt laut Bretherton et al. (1981) etwa ab einem Alter von 1;6. Dass ein Kind einen IST versteht und in bestimmten Kontexten auch produziert, bedeutet allerdings noch nicht, dass es beim Erzählen einer Geschichte ausgeprägte Anwendung von Theory of Mind zeigt und innere Zustände der Figuren ausdrückt. 


\section{Verben}

\subsection{Motivation zur Forschungsfrage}

Wenn ein Zusammenhang zwischen der Entwicklung lexikalischer und narrativer Fähigkeiten besteht, könnte dabei allen Wortarten eine vergleichbare Rolle zukommen. Vorstellbar sind jedoch auch wortartspezifische Unterschiede.

Wie genau Verben definiert werden, ist von der Perspektive abhängig. Rein syntaktisch kann ein Verb bestimmt werden als ein Wort, das ein Subjekt und je nach Verb auch ein oder mehrere Objekte bindet (Frawley, 1992). Als Prädikat ist das Verb der Kern des Satzes, der durch seine Argumentstellen ergänzt wird. Semantisch kann es definiert werden als ein Wort, das vor allem Ereignisse kodiert: „A cover term for states or conditions of existence (e.g., be sad), processes or unfoldings, (e.g., get sad), and actions or executed processes (e.g., sadden)" (Frawley, 1992:141).

Die Überlegung, dass besonders Verben entscheidend sind, kommt aus verschiedenen Gründen zustande. Verben bilden einen wesentlichen Bestandteil des Lexikons und sind wichtig für die Struktur und Organisation eines kohärenten Textes. Denn um eine kohärente Geschichte erzählen zu können, müssen Handlungen und Ereignisse sprachlich ausgedrückt werden können. Die sprachliche Bezeichnung von Handlungen erfolgt typischerweise durch Verben. Daneben können Verben auch Zustände wie bleiben oder liegen und Gefühle wie empfinden beschreiben.

Äußerungen wie will fressen, klettert hoch oder hat verjagt sind ohne Subjekt und Objekt zwar unvollständig, drücken aber Teile einer Geschichte wie Goal (will fressen), Attempt (klettert hoch) und Outcome (hat verjagt) aus. Fuchs, Katze oder Vogelnest sind ohne Verb nicht nur unvollständige Äußerungen, sondern erzählen gar keine Geschichte. Die Centrality of verbs (Zentralität von Verben) ist als Annahme in verschiedenen Theorien des Spracherwerbs etabliert. Wie eingeschränkt die sprachliche Ausdrucksfähigkeit ohne Verben wäre, schildern zum Beispiel Pence et al.: „Without verbs we are limited largely to commenting on the perceptual attributes and functions of the things around us. Verbs broaden our possibilities immensely" (Pence et al., 2005:64). Besonders aussagekräftig ist auch das folgende Zitat von Bloom über die Bedeutung von Verben:

As important as nouns are, [...] there has been the repeated finding that the verb is the real hero in determining what children learn about language structure [...] Verbs [...] reflect conceptual development (and) the semantics of the verbs that children learn have a mediating effect on language learning. (Bloom, 1978:1-2, zitiert in Golinkoff et al., 1995) 


\subsection{Verberwerb}

Für die Makrostruktur einer Geschichte ist vor allem die semantische Ebene ausschlaggebend. Für die Auswertung der $S S$ wird sowohl für Beispiel a) als auch das morphologische und syntaktische Fehler enthaltende Beispiel b) Goal als erfolgreich produziert betrachtet. In Beispiel b) sind die Fehler mit [*] markiert und die jeweiligen grammatisch korrekten Formen in Klammern nach einem Doppelpunkt ergänzt. ${ }^{3}$

a) Ungeduldig lauerte der hungrige Fuchs hinter dem Baum und beobachtete die Ziegenkinder, die er so sehr fressen wollte.

b) Ein hungriger Fuchs siehte [: sah] [*] den [: die] [*] Ziegenkinders [: Ziegenkinder] [*], die er <wollte so gerne auffressen> [: so gerne auffressen wollte] [*].

Bezogen auf die semantische Ebene erfordert der Verberwerb, sprachliche Formen (die Verben) mit den Bedeutungen und Konzepten, also der entsprechenden Repräsentation in der Welt, zu verknüpfen. Die Bedeutung eines Verbes erworben zu haben, umfasst auch das Wissen um die Valenz. So beinhaltet der (mentale) Lexikoneintrag des Verbs regnen, dass es avalent ist. Ebenso gehört es zum semantischen Wissen über das Verb geben, dass es trivalent ist ${ }^{4}$ und neben dem Agens die Theta-Rollen Patiens/Thema und Rezipient erfordert.

$\mathrm{Zu}$ den ersten Wörtern beziehungsweise wortähnlichen Formen, die ein Kind produziert, gehören zumeist onomatopoetische Ausdrücke und idiosynkratische Protowörter. Protowörter sind eine Form der kindlichen Wortproduktion, die im Verlauf der Entwicklung phonetische Konsistenz aufweist, aber durch Kontextgebundenheit charakterisiert ist. Echte referentielle Wörter wie zielsprachliche Verbformen werden ab dem zweiten Lebensjahr produziert. Nun werden Protowörter zunehmend dekontextualisiert, das heißt, das Kind löst sich vom situationsabhängigen Wortgebrauch (Kauschke, 2000).

Durch die interindividuelle Varianz beim Verberwerb kann nicht pauschal beantwortet werden, wie viele Verben Kinder beispielsweise mit 1;6 erworben haben. Allgemein gilt, dass der rezeptive, auch als passiv bezeichnete, Wortschatzerwerb dem produktiven, auch aktiv genannten, vorausgeht. Im Alter von eineinhalb Jahren ist die Differenz zwischen den Wörtern, die das Kind versteht, und denen, die es selbst produziert, sehr ausgeprägt (Clark, 2003).

3 Anmerkung: Die Beispiele sind erfunden.

4 Geben kann in bestimmten sprachlichen Kontexten auch ohne Dativobjekt gebraucht werden. Zum Beispiel in dem Satz: Anna gibt jeden Donnerstag Nachhilfestunden. 
Erst ab dem dritten Lebensjahr steigt die Verbproduktion an (Smith \& Sacks, 1990). Einige Verben, vor allem Bewegungsverben wie laufen und rennen, werden zwar bereits von unter zweijährigen Kindern verwendet, aber oft noch nicht verstanden, wenn sich diese Verben nicht auf sie selbst, sondern auf andere beziehen. Erst ab etwa 24 Monaten verstehen Kinder Bewegungsverben dann auch in diesen Kontexten (Huttenlocher, Smiley \& Ratner, 1985). In diesem Alter werden außerdem Mehrzweckverben wie machen erworben (Komor, 2008). Im Gegensatz zu Nomen werden Verben in der frühkindlichen Phase des Spracherwerbs nicht als Einwortäußerungen, sondern in Verbindung mit anderen Wörtern gebraucht. Daraus kann nach Kauschke (2002) ein Zusammenhang zwischen dem Erwerb von Verben und der syntaktischen Ebene abgeleitet werden.

Sogenannte Change Verbs (z.B. stellen) werden mit etwa 30 Monaten verstanden, wenn sie sich auf Tätigkeiten anderer beziehen (Huttenlocher et al., 1985). Nun werden auch vielseitigere und differenziertere Verben erlernt. Dazu gehören bereits erste Partikelverben, Auxiliare und Modalverben (Kauschke, 2002). Monolingualer und bilingualer Erstspracherwerb unterscheiden sich nicht in allen diesen Entwicklungsstadien. Der bilinguale Spracherwerb geht jedoch mit speziellen Herausforderungen und Besonderheiten einher. Das betrifft auch den Wortschatzerwerb, denn Bedeutungskonzepte sind nicht immer uneingeschränkt zwischen den Sprachen übertragbar.

Wenn bilinguale Kinder in den einzelnen Sprachen im Vergleich zu monolingualen weniger Input haben, ist es nicht verwunderlich, wenn sie bei einem Wortschatztest weniger Items in der Zielsprache korrekt benennen können. Wenn man jedoch beide Sprachen beachtet, ist festzustellen, dass bilinguale Kinder oft bereits mehr Konzepte als gleichaltrige monolingual aufwachsende Kinder erworben haben (Pearson \& Fernandez, 1994). Nicht alle Wörter werden gleichzeitig in beiden Sprachen gelernt.

\section{$4 \quad$ Forschungsfragen und Hypothesen}

\subsection{Forschungsfragen}

Die Fragestellungen sind in zwei Teile gegliedert. Der erste Teil adressiert beide Sprachen unabhängig voneinander. Im zweiten Teil wird anschließend der Zusammenhang zwischen den Sprachen betrachtet (intraindividuell). Um diese ergänzende Analyse zu ermöglichen, wurden bilinguale Probanden gewählt. 
I. Besteht, bei separater Betrachtung der beiden Sprachen Deutsch und Russisch, ein Zusammenhang zwischen dem produktiven Verbwortschatz und den narrativen Fähigkeiten $(S S, S C, I S T) ?^{5}$

1. Gibt es positive Korrelationen zwischen dem durch Sprachstandstests erfassten produktiven Verbwortschatz und den narrativen Fähigkeiten?

2. Liegt eine positive Korrelation zwischen der Anzahl der in den Narrativen produzierten Verben (auf Token- und auf Lemma-Ebene) und den narrativen Fähigkeiten vor?

Für I.1 und I.2 soll zudem jeweils geprüft werden, ob die Ergebnisse spezifisch für Verben sind, oder ob ähnliche Korrelationen zwischen dem produktiven Nomenwortschatz und den narrativen Fähigkeiten bestehen. ${ }^{6}$

II. Sind sprachübergreifende Zusammenhänge zwischen produktivem Verbwortschatz und narrativen Fähigkeiten festzustellen?

1. Ist der produktive Verbwortschatz der einzelnen Kinder in beiden Sprachen ähnlich ausgeprägt? ${ }^{7}$

2. Korreliert die $S S$ der einzelnen Kinder zwischen den beiden Sprachen?

3. Spiegelt sich das Verhältnis zwischen dem Verbwortschatz beider Sprachen (Dominanz in einer Sprache vs. ausgeglichener Verbwortschatz) in Unterschieden zwischen der $S S$ wider?

\subsection{Hypothesen}

Die dieser Studie wesentlich zugrundeliegende Hypothese ist, dass aus den in Kapitel 3.1 erörterten Gründen möglicherweise speziell Verben für die narrativen Fähigkeiten relevant sind. Es wird allerdings vermutet, dass der Verbwortschatz bei altersgemäßer Entwicklung der lexikalischen Fähigkeiten ab einer gewissen Entwicklungsstufe einen weniger ausgeprägten Effekt hat. $\mathrm{Zu}$ erwarten ist

5 Der Begriff narrative Fähigkeiten bezieht sich in allen in diesem Kapitel aufgestellten Fragestellungen auf die drei Makrostrukturkomponenten SS, SC, IST.

6 Da Verb- und Nomenwortschatz in den untersuchten Daten positiv miteinander korrelieren (der Korrelationskoeffizient beträgt in den Probandengruppen durchschnittlich etwa 0,5; bei den 6-Jährigen im Russischen sogar 0,78), wäre es möglich, dass die Ergebnisse auf einen generellen Zusammenhang zwischen produktiven lexikalischen Fähigkeiten und narrativen Fähigkeiten hinweisen. Der Vergleich mit dem Nomenwortschatz dient dem Zweck, einschätzen zu können, ob es empirisch belegt Grund zu der Annahme gibt, dass speziell der Verberwerb eine entscheidende Rolle für die Entwicklung der narrativen Fähigkeiten spielt.

7 Der Vergleich erfolgt hierbei in Relation zur jeweiligen Probandengruppe und beinhaltet somit keinen absoluten Vergleich des Verbwortschatzes zwischen den Sprachen. 
außerdem, dass ein stärkerer Zusammenhang zwischen dem Verbwortschatz und den Makrostrukturkomponenten IST und SS besteht als zwischen Verbwortschatz und SC. Dies wird wegen des von Gagarina (2016) beschriebenen Kontinuums von $I S T$ als am meisten sprachabhängig über $S S \mathrm{zu}$ der am wenigsten sprachabhängigen Komponente $S C$ - angenommen.

\section{$5 \quad$ Methode}

\subsection{Probanden}

Bei den Probanden handelt es sich um in Berlin lebende deutsch-russisch bilinguale Kinder, von denen einige, aber nicht alle, in Deutschland geboren sind. Probanden, bei denen Russisch die Muttersprache beider Eltern ist, haben oft erst mit Eintritt in die Kindertagesstätte begonnen, die deutsche Sprache zu erwerben. Die individuellen Bedingungen des bilingualen Spracherwerbs und die Kontaktdauer mit der deutschen Sprache variieren also zwischen den Probanden.

Die Vorschüler wurden aus verschiedenen Berliner Kindertagesstätten im Rahmen der longitudinalen BIVEM-Studie ${ }^{8}$ rekrutiert und sind teils zum ersten Mal, teils bereits zum zweiten Mal mit MAIN und den Sprachstandstests getestet worden. ${ }^{9}$ Die Erst- und Drittklässler stammen aus einer staatlichen bilingualen Schule, wo der Unterricht in deutscher und russischer Sprache stattfindet. Bei ihnen wurde jeweils zum ersten Mal eine Geschichte mit MAIN elizitiert.

Die Gruppe der Erstklässler wurde für die Untersuchung der Fragestellungen I.1 und II deshalb noch einmal unterteilt, weil der deutsche Wortschatztest, der durchgeführt wurde, unterschiedliche Items für 6- und 7Jährige enthält (Genaueres dazu unter Kapitel 5.2.1). Für die Fragestellung I.2 können die Erstklässler als eine Gruppe betrachtet werden, da hierfür die Verbproduktion direkt in den Narrativen analysiert wurde. Genaue Angaben zu Anzahl und Durchschnittsalter der Kinder sind in der Tabelle 1 für die jeweiligen Probandengruppen zusammengestellt.

8 http://www.leibniz-zas.de/bivem.html

9 Vorschüler bedeutet hierbei nicht, dass die Kinder die Vorschule besuchen, sondern, dass sie sich im vorschulischen Alter befinden. Einer der 21 Probanden dieser Gruppe ist zum dritten Mal getestet worden. 
Tabelle 1: Übersicht über die Probandengruppen

\begin{tabular}{|c|c|c|c|c|}
\hline \multicolumn{2}{|c|}{ Probanden-Gruppe } & \multirow{3}{*}{$\begin{array}{l}\begin{array}{l}\text { Anzahl } \\
(N)\end{array} \\
21\end{array}$} & \multicolumn{2}{|c|}{$\begin{array}{l}\text { Durchschnittsalter } \\
\text { in Monaten }(S D) \\
\text { ne } \\
\text { zum Zeitpunkt der Elizitierung } \\
\quad \text { der Narrative }\end{array}$} \\
\hline & Deutsch & & $45(5.8)$ & \\
\hline \multirow[t]{2}{*}{ Vorschüler } & & & & $31-52$ \\
\hline & Russisch & 18 & $45(6.0)$ & \\
\hline \multirow{2}{*}{$\begin{array}{l}\text { Erstklässler } \\
\text { Forschungsfra } \\
\text { ge I.1 }\end{array}$} & $\begin{array}{l}\text { 6-Jährige } \\
\text { (Deutsch und Russisch) }\end{array}$ & 21 & $80(2.0)$ & $76-83$ \\
\hline & $\begin{array}{l}\text { 7-Jährige } \\
\text { (Deutsch und Russisch) }\end{array}$ & 23 & $86(2.4)$ & $82-92$ \\
\hline \multirow{2}{*}{$\begin{array}{l}\text { Erstklässler } \\
\text { Forschungsfra } \\
\text { ge I.2 }\end{array}$} & Deutsch & 44 & $83(3.8)$ & \multirow[b]{2}{*}{$76-92$} \\
\hline & Russisch & 26 & $82(4.0)$ & \\
\hline Drittklässler & Deutsch und Russisch & 22 & $111(8.0)$ & $95-126$ \\
\hline \multicolumn{5}{|c|}{$\begin{array}{l}\text { Anmerkung: Die Angaben sind zum Teil für die Sprachen einzeln aufgeführt, da von manchen Probanden nur } \\
\text { in einer Sprache vollständige Daten vorliegen. Zum Beispiel haben in der Gruppe der Vorschüler drei der } \\
\text { Kinder keine Geschichte auf Russisch produziert. Bei der Gruppe der Erstklässler lag die morphologische } \\
\text { Kodierung nicht für alle Probanden in beiden Sprachen vor. }\end{array}$} \\
\hline
\end{tabular}

\subsection{Design}

\subsubsection{Datenerhebung}

Mittels der Bildergeschichten von MAIN (Gagarina et al. 2012), das im Rahmen der COST Action IS0804 entwickelt wurde, wurden in beiden Sprachen Narrative elizitiert. Für die Erhebung der in dieser Studie verwendeten Daten wurde der Modus Telling genutzt. Bevor die Kinder selbst eine Geschichte produziert haben, wurde ihnen eine Model Story vorgelesen (Dog oder Cat). Anschließend wurde eine ähnliche, parallel konzipierte Bildergeschichte gezeigt, die von den Kindern aus einem selbst gewählten Umschlag ausgepackt und versteckt angeschaut wurde. Jedes Kind hat jeweils eine Geschichte auf Russisch und eine auf Deutsch erzählt. Einmal Baby Goats und in der anderen Sprache Baby Birds. ${ }^{10}$ Zwischen diesen beiden Testungen mit MAIN lag eine Zeitspanne von wenigstens einigen Tagen, in den meisten Fällen mindestens eine Woche. Die Narrative wurden nach dem CHAT-Format transkribiert und mit Hilfe des Programms CLAN

10 Welche Geschichte in welcher Sprache erzählt wurde, ist zwischen den Probanden ausgeglichen, das heißt, etwa die Hälfte der Kinder hat $B B$ auf Russisch und $B G$ auf Deutsch erzählt, für die andere Hälfte war es umgekehrt. 
(MacWhinney, 2000) morphologisch kodiert, wodurch eine Frequenzanalyse der Wortarten und Wortformen ermöglicht wird (s. Anhang für Beispiele des CLANOutputs).

Für die deutsche Sprache wurden zwei verschiedene Testverfahren zur Erfassung des Wortschatzes verwendet: Bei den Vorschülern PDSS, ausgeschrieben Patholinguistische Diagnostik bei Sprachentwicklungsstörungen (Kauschke \& Siegmüller, 2010), und bei den Erstklässlern der Wortschatz- und Wortfindungstest für 6- bis 10-Jährige (WWT, Glück, 2011). Sowohl die Vorschüler als auch die Erstklässler haben für das Russische den Sprachstandstest Russisch für mehrsprachige Kinder (SRUK, Gagarina, Klassert \& Topaj, 2010) absolviert.

Der Untertest Wortproduktion der PDSS umfasst für Verben und Nomen jeweils 20 Items. Die Verben sind: schwimmen, tragen, sitzen, retten, gähnen, schieben, kämpfen, spucken, öffnen, niesen, füttern, tauchen, wandern, kneifen, grüßen, krabbeln, messen, pflücken, wiegen, zaubern.

Beim WWT handelt es sich um ein Testverfahren zur Diagnostik semantisch-lexikalischer Fähigkeiten, welches für Kinder im Alter von 5;6 bis 10;11 Jahren konzipiert wurde. Es gibt verschiedene Varianten zwischen denen gewählt werden kann. Mit den Probanden, die Teil dieser Studie sind, wurde je nach Alter Kurzform 1 (5;6 - 6;11 Jahre) oder Kurzform 2 (7;0 - 8;11 Jahre) durchgeführt, die jeweils 10 Verben beinhalten. Kurzform 1 (KF 1) und 2 (KF 2) unterscheiden sich hinsichtlich des Schwierigkeitsgrads voneinander, weshalb die Ergebnisse der 6-Jährigen nicht unmittelbar mit den Ergebnissen der 7-Jährigen verglichen werden können.

Die Verben in KF 1 sind: wiegen, abtrocknen, ziehen, brüllen/gähnen, abschleppen/abtransportieren, jonglieren, verblühen/verwelken, wehen/flattern, verbeugen/verneigen, stricken. Die Verben in KF 2: ziehen, brüllen/gähnen, abschleppen/abtransportieren, jonglieren, verblühen/verwelken, wehen/flattern, verbeugen/verneigen, stricken, entgleisen, reiben/hobeln/raspeln. ${ }^{11}$

Es sind also acht der Verben gleich, jedoch sind gerade die beiden Verben entgleisen und reiben/hobeln/raspeln von kaum einem der Probanden produziert worden. Für den Vergleich in Relation zur Altersgruppe existieren Normwerte (TWerte sowie Prozentränge). Der Entwicklungsstand des Verbwortschatzes im Verhältnis zur (monolingualen) Norm ist jedoch nicht Gegenstand dieser Studie. Stattdessen bezwecken die Untersuchungen, den Zusammenhang zwischen den narrativen Fähigkeiten und dem absoluten Stand des Verbwortschatzes zu erforschen. Daher wird die Gruppe der Erstklässler für die Fragestellungen I.1 und II aufgeteilt, sodass nur die Kinder, bei denen der gleiche Test durchgeführt wurde, direkt miteinander verglichen werden.

11 Die Schrägstriche bedeuten, dass beide oder mehrere Wörter als korrekt gewertet werden. 
Der Sprachstandstest Russisch (SRUK) wurde eigens für mehrsprachige Kinder entwickelt. Für das Forschungsanliegen dieser Studie wurde der Untertest 1 Lexikon Nomen und Verben einbezogen. Für Nomen und Verben gibt es jeweils 26 Items. Die Verben lauten: sidet' (sitzen), pilit' (sägen), spat' (schlafen), bit " (schlagen), tancevat' (tanzen), goret' (brennen), lajat' (bellen), razbit'/razbivat' (kaputtmachen/zerschlagen), čitat' (lesen), lit' (gießen), risovat' (malen), prolit'/prolivat' (verschütten), jest'/kušat' (essen), upast'/padat' (herunterfallen/ fallen), letet' (fliegen), svistet' (pfeifen), pit' (trinken), šeptat ' (flüstern), bežat' (laufen), ščipat' (kneifen), sažat' (anpflanzen), šit' (nähen), sosat' (saugen), ukrast'/krast' (klauen/stehlen), laskat '(streicheln), (raz)rezát' (schneiden).

Zusätzlich zu den oben genannten Tests wurden die durch MAIN elizitierten Narrative hinsichtlich des Verbgebrauchs analysiert, was als weiteres $\mathrm{Ma}$ des produktiven Verbwortschatzes dienen soll ${ }^{12}$. Für diese Untersuchung wurde die Anzahl der insgesamt produzierten Verben in den Narrativen ermittelt. Darüber hinaus wurde die Anzahl der unterschiedlichen Verblemmata, die produziert wurden, erfasst. Somit kann zwischen der rein quantitativ analysierten Verbproduktion (Anzahl der Tokens) und der Verbproduktion auf LemmataEbene differenziert werden.

Die Verben sein, werden und haben erhalten bei der Kodierung jeweils separate Einträge, wenn sie als Kopula- oder Auxiliarverb gebraucht werden. Für die Bestimmung der Anzahl der verschiedenen Lemmata wird bei diesen Verben nicht zwischen Kopula und Auxiliar unterschieden. In dieser Studie werden Partikelverben wie herunterfallen und wegfliegen als separate Lemmata betrachtet - unabhängig davon, ob die gleichen Verbstämme ohne diese Partikeln zusätzlich verwendet wurden (hier: fallen beziehungsweise fliegen). Analog gilt für Präfixverben wie beispielsweise verjagen, dass sie als auch dann als separates Lemma gezählt werden, wenn zusätzlich das entsprechende Verb ohne Präfix auftritt (hier: jagen). Diese Entscheidung wurde aus dem Grund getroffen, dass die Verwendung von Partikel- und Präfixverben, also komplexeren Verben, von einem differenzierteren Verbwortschatz zeugt. Bei den Nomen wurden Diminutivbildungen als eigenständige Lemmata gezählt.

\subsubsection{Vorgehensweise bei der statistischen Auswertung}

12 Anmerkung: Der Verbgebrauch in den Narrativen ist kein absolut repräsentatives Maß für den Entwicklungsstand des produktiven Verbwortschatzes. Jedoch zeigt die Forschung, dass Narrative als Sprachsample aussagekräftig für den sprachlichen Entwicklungsstand sind. 
Zur Auswertung der Makrostruktur ist noch anzumerken, dass die Korrelationstests für die Komponente $S C$ anhand der Anzahl der vollständigen Episoden $(G A O)$ erfolgt, da eine GAO-Sequenz in MAIN die höchste Stufe der Komplexität darstellt. Von den Vorschülern haben allerdings nur insgesamt zwei Kinder bereits eine GAO-Sequenz produziert. Deshalb wurde für diese Probandengruppe die Summe aus $G A O, G A$ und $G O$ gebildet. $G A$ und $G O$ werden als abgekürzte Episoden betrachtet (Gagarina et al. 2012) und weisen schon eine stärker ausgeprägte Komplexität auf als beispielswese ein einzelnes A in einer Episode, weshalb sie für die Gruppe der Vorschüler mitberücksichtigt werden. Die IST werden für die Korrelationsberechnungen nach der Länge der produzierten Geschichte, das heißt der Gesamtzahl der Tokens, genormt und in Prozent angegeben. Das ist einerseits sinnvoll, da in eine längere Geschichte auch mehr IST eingebracht werden können und auch Wiederholungen des gleichen IST gezählt werden. Nicht außer Acht sollte jedoch gelassen werden, dass diese Normierung auch dazu führt, dass ein Kind, welches eine sehr kurze Geschichte erzählt und einen oder zwei $I S T$ produziert, einen verhältnismäßig hohen Prozentsatz an IST aufweisen wird.

Hinführend $\mathrm{zu}$ Kapitel 6. Ergebnisse soll nun die strukturelle Vorgehensweise der Untersuchung erläutert werden, damit diese besser nachvollzogen werden kann. Der Aufbau des Ergebnisteils richtet sich nach der Gliederung der in Kapitel 4 formulierten Fragestellungen: Im ersten Teil (bestehend aus I.1 und I.2) werden die Zusammenhänge zwischen dem Verberwerb und den narrativen Fähigkeiten im Deutschen und im Russischen unabhängig voneinander untersucht. Der zweite Teil verfolgt das Ziel, Vergleiche zwischen den Sprachen zu ziehen. Hier werden die narrativen Fähigkeiten und der Verbwortschatz der Probanden in L1 (Russisch) und L2 (Deutsch) in Relation zueinander untersucht. Die Auswertung der Daten für die Altersgruppen und Sprachen erfolgt stets in der gleichen Reihenfolge: Vorschulkinder, Erstklässler, Drittklässler ${ }^{13}$; jeweils beginnend mit Deutsch. Für die Berechnungen wird das Programm R verwendet (R Core Team, 2014).

I. Analyse des Zusammenhangs zwischen Verberwerb und narrativen Fähigkeiten bei separater Betrachtung der Sprachen

Für jede Probandengruppe werden zunächst die Ergebnisse für Verben dargelegt. Um herauszufinden, ob diese Ergebnisse spezifisch für Verben sind, wird jeweils im Anschluss der Vergleich mit den Nomen herangezogen. Aufgrund dieses

13 Sofern für die Fragestellung alle diese Altersgruppen untersucht worden sind. Nicht alle Analysen konnten für alle Gruppen durchgeführt werden. 
doppelten/vierfachen Testens wird ein adjustiertes Signifikanzniveau von 0.025 für I.1 und 0.0125 für I.2 angelegt.

1. Untersuchung auf Korrelation zwischen $S S, S C, I S T$ und dem produktiven Verbwortschatz; ausgewertet nach Altersgruppe und Sprache für die Probandengruppen: Vorschüler (deutsch $N=21$, russisch $N=18$ ), Erstklässler: 6-Jährige $(N=21)$ und 7-Jährige $(N=23)$.

2. Untersuchung auf Korrelation zwischen $S S, S C$, IST und der Verbproduktion in den Narrativen; ausgewertet nach Altersgruppe und Sprache für die Probandengruppen: Erstklässler (Deutsch: $N=44$, Russisch: $N=26$ ) und Drittklässler $(N=22)$

II. Zusammenhänge zwischen den Sprachen beim Verbwortschatz und der Makrostrukturkomponente $S S$

Untersuchte Probandengruppe: Vorschüler $\left(N=18^{14}\right)$

1. Vergleich des Verbwortschatzes zwischen den Sprachen

2. Test auf Korrelation zwischen der $S S$ in beiden Sprachen

3. Test auf Unterschiede bei der Abweichung zwischen der $S S$ in Deutsch und Russisch, in Abhängigkeit vom Verhältnis des Verbwortschatzes beider Sprachen

\section{Ergebnisse}

\subsection{Teil I}

\subsubsection{Ergebnisse zu Forschungsfrage I.1}

Zur Bearbeitung der Forschungsfrage I.1 wurde untersucht, ob Korrelationen zwischen den drei Makrostrukturkomponenten und dem produktiven Verbwortschatz bestehen. ${ }^{15}$

14 Da drei der 21 Probanden der Vorschüler-Gruppe im Russischen keine Geschichte produziert haben, ist der Vergleich zwischen den Sprachen nur für 18 Vorschüler möglich.

15 Ein parametrischer Test (Pearson's Product Moment Correlation) setzt Normalverteilung beider betrachteter Datensätze als Bedingung voraus. Als Test auf Normalverteilung wurde jeweils der Shapiro-Wilk-Test durchgeführt. Hat sich gezeigt, dass nicht für beide Datensätze eine Normalverteilung angenommen werden kann, so wurde statt der Pearson's Product Moment Correlation ein nicht-parametrischer Test angewendet (Kendalls Tau). 
Tabelle 2: Vorschulkinder Deutsch $(N=21)$ : Korrelationstests (Angabe von Pearson's $r$ / Kendalls tau $\left(r_{\tau}\right)$ und den $p$-Werten)

\begin{tabular}{lll}
\hline MAKROSTRUKTUR & VERBEN & NOMEN \\
\hline$S S$ & $r(19)=.39$ & $r_{\tau}=.34$ \\
& $p=.08$ & $p=.05^{16}$ \\
\hline$G A O / G A / G O$ & $r_{\tau}=.17$ & $r_{\tau}=.10$ \\
& $p=.37$ & $p=.61$ \\
\hline$I S T$ in $\%$ & $r_{\tau}=.21$ & $r_{\tau}=.23$ \\
& $p=.23$ & $p=.19$ \\
\hline
\end{tabular}

Bei den Vorschülern liegen im Deutschen weder für Verben noch für Nomen signifikante Korrelationen vor. Für $S S$ deuten die Korrelationskoeffizienten am ehesten auf mäßig positive Korrelationen hin, allerdings erreichen auch diese nicht das geforderte Signifikanzniveau von .025 . Bei Russisch $(N=18)$ liegen weder signifikante Korrelationen noch Tendenzen vor.

Tabelle 3: Erstklässler: 6-Jährige Deutsch $(N=21)$ : Korrelationstests (Angabe von Pearson's $r$ / Kendalls tau $\left(r_{\tau}\right)$ und den $p$-Werten)

\begin{tabular}{lll}
\hline MAKROSTRUKTUR & VERBEN & NOMEN \\
\hline$S S$ & $r(19)=.11$ & $r(19)=.34$ \\
& $p=.64$ & $p=.13$ \\
\hline$G A O$ & $r_{\tau}=. .01$ & $r_{\tau}=.31$ \\
& $p=.97$ & $p=.09$ \\
\hline$I S T$ in $\%$ & $r(19)=.05$ & $r(19)=.26$ \\
& $p=.85$ & $p=.26$ \\
\hline
\end{tabular}

Tabelle 4: Erstklässler: 6-Jährige Russisch $(N=21)$ : Korrelationstests (Angabe von Pearson's $r$ / Kendalls tau $\left(r_{\tau}\right)$ und den p-Werten)

\begin{tabular}{lll}
\hline MAKROSTRUKTUR & VERBEN & NOMEN \\
\hline$S S$ & $r(19)=.29$ & $r(19)=.36$ \\
& $p=.20$ & $p=.11$ \\
\hline$G A O$ & $r_{\tau}=.21$ & $r_{\tau}=.25$ \\
& $p=.26$ & $p=.17$ \\
\hline$I S T$ in $\%$ & $r(19)=.00$ & $r(19)=.04$ \\
& $p=.99$ & $p=.85$ \\
\hline
\end{tabular}

16 Abgerundet. 
Für beide Sprachen liegen bei der Gruppe der 6-Jährigen keine signifikanten Korrelationen vor und Tendenzen deuten sich eher für Nomen als für Verben an. ${ }^{17}$

Bei den 7-Jährigen liegen weder für Deutsch noch für Russisch positive signifikante Korrelationen vor. Bei Deutsch gibt es eine signifikante negative Korrelation zwischen $I S T$ in $\%$ und Verben $(r(21)=-.50, p=.02)$.

\subsubsection{Ergebnisse zu Forschungsfrage I. $2^{18}$}

Die meisten der hier zu vergleichenden Daten sind nicht normalverteilt. Deshalb wird nun für alle Korrelationen in diesem Abschnitt ein nicht-parametrischer Test verwendet (Kendalls tau).

Tabelle 5: Erstklässler: Deutsch $(N=44)^{19}$ : Korrelationstests (Angabe von Kendalls tau und den $p$-Werten darunter)

\begin{tabular}{lllll}
\hline MAKRO- & \multicolumn{2}{c}{ VERBEN } & \multicolumn{2}{c}{ NOMEN } \\
STRUKTUR & Tokens & Lemmata & Tokens & Lemmata \\
\hline \multirow{2}{*}{$S S$} & $\mathbf{. 3 9}$ & $\mathbf{. 4 6}$ & $\mathbf{. 3 3}$ & $\mathbf{. 4 2}$ \\
& $\boldsymbol{p}<.01$ & $\boldsymbol{p}<. \mathbf{0 1}$ & $\boldsymbol{p}<. \mathbf{0 1}$ & $\boldsymbol{p}<. \mathbf{0 1}$ \\
\hline \multirow{2}{*}{$G A O$} & .27 & $\mathbf{4 0}$ & .20 & $\mathbf{. 3 9}$ \\
& $p=.03$ & $\boldsymbol{p}<. \mathbf{0 1}$ & $p=.10$ & $\boldsymbol{p}<. \mathbf{0 1}$ \\
\hline \multirow{2}{*}{$I S T$ in $\%$} & .03 & .01 & -.01 & .03 \\
& $p=.80$ & $p=.91$ & $p=.93$ & $p=.78$ \\
\hline
\end{tabular}

Es gibt signifikant positive Korrelationen zwischen den Verb-Tokens und $S S$ sowie auch zwischen den Verb-Lemmata und $S S / G A O$. Ähnlich ist es für Nomen, jedoch sind diese weniger stark ausgeprägt.

17 Die deutschen Test-Items für Nomen setzen sich beim WWT aus Objekten und KategorieWörtern zusammen. Für diese Untersuchung sind die gemittelten Werte herangezogen worden. Betrachtet man nur die Nomen, die Objekte bezeichnen, so lässt sich hier eine marginal signifikante positive Korrelation zu SS feststellen $(r(19)=0.44, p<.05)$.

18 Vorbemerkung: Verb-Tokens und -Lemmata in Prozent ergaben für $S S$ keine positiven Korrelationen. Da es bei allen getesteten Probandengruppen keine signifikant positiven - oder überhaupt nennenswert positiven - Korrelationen mit den genormten Werten für Verb-Tokens und -Lemmata gab, sind diese Werte nicht aufgeführt.

19 Da hier nur die Narrative ausgewertet werden, besteht kein Anlass, die Gruppe der Erstklässler aufzuteilen. 
Tabelle 6: Erstklässler: Russisch $(N=26)$ : Korrelationstests (Angabe von Kendalls tau und den $p$-Werten darunter)

\begin{tabular}{lllll}
\hline MAKRO- & \multicolumn{2}{c}{ VERBEN } & \multicolumn{2}{c}{ NOMEN } \\
STRUKTUR & Tokens & Lemmata & Tokens & Lemmata \\
\hline \multirow{2}{*}{$S S$} & .36 & $\mathbf{. 4 1}$ & .36 & .22 \\
& $p=.02$ & $\boldsymbol{p}<.01$ & $p=.02$ & $p=.16$ \\
\hline \multirow{2}{*}{ GAO } & .29 & .29 & .13 & .38 \\
& $p=.08$ & $p=.08$ & $p=.43$ & $p=.03$ \\
\multirow{2}{*}{$I S T$ in $\%$} & -.14 & -.06 & -.01 & -.11 \\
& $p=.33$ & $p=.66$ & $p=.95$ & $p=.46$ \\
\hline
\end{tabular}

Die Anzahl der Verb-Lemmata korreliert auch im Russischen positiv mit SS. Marginal signifikant positive Korrelationen bestehen außerdem zwischen $S S$ und den Verb- sowie Nomen-Tokens.

Tabelle 7: Drittklässler: Deutsch $(N=22)$ : Korrelationstests (Angabe von Kendalls tau und den $p$-Werten darunter)

\begin{tabular}{lllll}
\hline MAKRO- & \multicolumn{2}{c}{ VERBEN } & \multicolumn{2}{c}{ NOMEN } \\
STRUKTUR & Tokens & Lemmata & Tokens & Lemmata \\
\hline \multirow{2}{*}{$S S$} & $\mathbf{. 5 4}$ & $\mathbf{. 4 6}$ & $\mathbf{. 5 2}$ & $\mathbf{. 5 6}$ \\
& $\boldsymbol{p}<. \mathbf{0 1}$ & $\boldsymbol{p}<. \mathbf{0 1}$ & $\boldsymbol{p}<. \mathbf{0 1}$ & $\boldsymbol{p}<. \mathbf{0 1}$ \\
\hline \multirow{2}{*}{$G A O$} & .13 & .05 & .28 & .20 \\
& $p=.48$ & $p=.79$ & $p=.13$ & $p=.28$ \\
\multirow{2}{*}{$I S T$ in $\%$} & .22 & $\mathbf{0 . 4 9}$ & .29 & .19 \\
& $p=.16$ & $\boldsymbol{p}<. \mathbf{0 1}$ & $p=.06$ & $p=.24$ \\
\hline
\end{tabular}

Es gibt signifikant positive Korrelationen zwischen $S S$ und den Verben und Nomen. IST in \% korreliert zudem mit der Anzahl der Verb-Lemmata.

Tabelle 8: Drittklässler: Russisch $(N=22)$ : Korrelationstests (Angabe von Kendalls tau und den $p$-Werten darunter)

\begin{tabular}{lllll}
\hline MAKRO- & \multicolumn{2}{c}{ VERBEN } & \multicolumn{2}{c}{ NOMEN } \\
STRUKTUR & Tokens & Lemmata & Tokens & Lemmata \\
\hline \multirow{2}{*}{$S S$} & $\mathbf{. 5 0}$ & $\mathbf{. 5 4}$ & .35 & .10 \\
& $\boldsymbol{p}<.01$ & $\boldsymbol{p}<.01$ & $p<.05$ & $p=.55$ \\
\hline \multirow{2}{*}{$G A O$} & .42 & .40 & .16 & -.07 \\
& $p<.05$ & $p<.05$ & $p=.38$ & $p=.72$ \\
\multirow{2}{*}{$I S T$ in $\%$} & .20 & .14 & .26 & .17 \\
& $p=.21$ & $p=.39$ & $p=.998$ & $p=.28$ \\
\hline
\end{tabular}


Nur die Korrelationen zwischen SS und Verben erreichen das geforderte Signifikanzniveau von 0.0125 .

\subsection{Teil II}

Für diesen Teil wurden die Daten der Vorschulgruppe analysiert $(N=18)$.

\subsubsection{Vergleich des Verbwortschatzes zwischen den Sprachen}

Damit der russische Wortschatztest (SRUK) mit dem deutschen verglichen werden kann, wurden Mittelwerte und Standardabweichungen für Verben, Nomen und Gesamtwortschatz (Nomen und Verben) berechnet, anhand derer eine Einteilung in drei Gruppen vorgenommen wurde: Gruppe 1: ab einer SD unter dem Mittelwert ${ }^{20}$, Gruppe 2: Spanne zwischen Gruppe 1 und 3, Gruppe 3: ab einer SD über dem Mittelwert.

Tabelle 9: Vorschüler: $(N=18)$. Übersicht über die Gruppeneinteilung. Die angegeben Werte sind die erzielten Punktzahlen beim Sprachstand Deutsch (PDSS) und Russisch (SRUK).

\begin{tabular}{lll}
\hline & $\begin{array}{l}\text { VERBEN- } \\
\text { DEUTSCH }\end{array}$ & $\begin{array}{l}\text { VERBEN- } \\
\text { RUSSISCH }\end{array}$ \\
\hline Mittelwert $(S D)$ & $3.71(2.07)$ & $10.52(4.59)$ \\
\hline Gruppe 1 & $0-2$ & $0-6$ \\
\hline Gruppe 2 & $3-5$ & $7-14$ \\
\hline Gruppe 3 & $6-20$ & $15-26$ \\
\hline
\end{tabular}

Nur 7 der 18 Probanden sind nach dieser Aufteilung in beiden Sprachen in der gleichen Gruppe. Demnach verfügen etwa 39\% über einen ähnlich weit entwickelten Verbwortschatz im Deutschen und Russischen (je im Verhältnis zur untersuchten Probandengruppe). 61\% (11 Probanden) sind in einer der Sprachen im Verhältnis besser, davon ist ein Proband in der einen Sprache in der Gruppe

20 Die Werte sind auf ganze Zahlen gerundet, da bei dem Wortschatztest nur volle Punktzahlen zu vergeben sind. Ist beispielsweise die obere Grenze von Gruppe 1 5.9, so wird auf 6 gerundet. Die Untergrenze von Gruppe 2 liegt dann bei der nächsthöheren ganzen Zahl (in diesem Fall 7). Analog gilt für Gruppe 3, dass, wenn die untere Grenze bei z. B. 15,1 liegt, auf 15 gerundet wird. Die Obergrenze von Gruppe 2 wird dann auf die nächstkleinere ganze Zahl festgelegt. Dadurch sind die Grenzen von Gruppe 2 etwas enger als plus/minus eine Standardabweichung. Alternativ hätten die Grenzen für Gruppe 1 und 3 jeweils enger gesetzt werden können, wenn Gruppe 2 als Ausgangspunkt gewählt wird und dann für Gruppe 1 die nächstkleinere ganze Zahl als Obergrenze und für Gruppe 3 die nächsthöhere ganze Zahl als Untergrenze festgesetzt worden wäre. Dies hätte zur Folge gehabt, dass fast alle Kinder in die mittlere Gruppe eingestuft worden wären. 
mit den höchsten und in der anderen Sprache in der Gruppe mit den niedrigsten Punktzahlen.

\subsubsection{Vergleich der SS zwischen den Sprachen}

Der Vergleich der Makrostruktur zwischen den Sprachen hingegen hat ergeben, dass die Abweichungen hier weniger stark als beim Wortschatz ausgeprägt sind. Die $S S$ im Deutschen und Russischen korreliert positiv miteinander $(r(16)=.59$, $p<.01)$.

\subsubsection{Zusammenhang von Unterschieden zwischen den Sprachen: Verbwortschatz und $S S$}

Gibt es bei den Probanden, deren Verbwortschatz zwischen den Sprachen ausgeglichener ist, auch geringere Abweichungen zwischen der $S S$ in der russischen und der deutschen Erzählung?

Anhand der Ergebnisse von 6.2.1 wurde die Gruppe für diese Fragestellung in zwei Subgruppen geteilt: (1) ausgeglichener Verbwortschatz $(\mathrm{N}=7)$ und (2) Dominanz in einer Sprache $(\mathrm{N}=11)$. Mit Hilfe eines Wilcoxon rank sum test wurde geprüft, ob ein Unterschied zwischen diesen beiden Subgruppen hinsichtlich der Abweichung zwischen den Sprachen bei der SS besteht. Der Test hat ergeben, dass Subgruppe (1) keine geringeren Abweichungen bei der $S S$ hat. Die mittlere Abweichung der Subgruppe (1) betrug 2.43, die bei Subgruppe (2) 1.09. Der Unterschied ist nicht signifikant $(\mathrm{W}=54.5, p=.14)$.

\section{$7 \quad$ Zusammenfassung und Diskussion}

Im Rahmen dieser Studie wurde der Zusammenhang zwischen der Makrostruktur elizitierter Narrative - SS, SC und IST (elizitiert und ausgewertet nach MAIN, Gagarina et al., 2012) - und dem produktiven Verbwortschatz bei deutsch-russisch bilingualen Kindern in beiden Sprachen untersucht. Als Maß für den Verbwortschatz wurden sowohl Wortschatztests (PDSS, WWT, SRUK) als auch die Verbproduktion direkt in den Narrativen berücksichtigt. Es wurden drei verschiedene Altersgruppen betrachtet: 21 Kinder im vorschulischen Alter (3;9), 44 Erstklässler (6;11) und 22 Drittklässler (9;3).

Die Frage, ob der Verbwortschatz einen bedeutenden Faktor für die Entwicklung der narrativen Fähigkeiten darstellt, kann nicht eindeutig beantwortet werden. Es wurden einige positive Korrelationen gefunden, jedoch ist nicht für alle Altersgruppen und Sprachen ein systematisches Muster erkennbar. Zudem ist bei der Interpretation der Ergebnisse zu beachten, dass Korrelationen nicht als Beleg für kausale Zusammenhänge betrachtet werden 
dürfen. Sie können aber Anhaltspunkte darüber liefern, wie sich verschiedene Aspekte zueinander verhalten. Wenn keine Korrelation besteht, kann zudem ein linearer Zusammenhang ausgeschlossen werden. Daraus, dass in dieser Studie in mehreren Fällen keine signifikante Korrelation gefunden wurde, lässt sich folglich ableiten, dass der Verbwortschatz (wie er in diesem Rahmen gemessen wurde) zumindest nicht der einzige maßgeblicher Faktor sein kann.

Entgegen der Hypothese wurden keine signifikanten positiven Korrelationen zwischen dem produktiven Verbwortschatz (erfasst durch Sprachstandstests) und der Makrostruktur in den elizitierten Narrativen gefunden. Für alle untersuchten Probandengruppen galt hier, dass sich bei Russisch nicht einmal Tendenzen abbildeten. Somit konnte kein verbspezifischer Effekt gezeigt werden.

Dass bei Deutsch zum Teil (bei den 7-Jährigen) negative Korrelationen zwischen IST in Prozent und Verben (signifikant) sowie zwischen IST in Prozent und Nomen (marginal signifikant) vorlagen, ist dadurch zu erklären, dass in sehr kurzen Geschichten bereits durch wenige IST ein hoher Prozentwert erreicht wird und sehr kurze Geschichten wiederum tendenziell eher von einem kleineren Wortschatz zeugen. Es kann vermutet werden, dass mit dem absoluten IST Maß eher ein positiver Zusammenhang gefunden worden wäre.

Im Hinblick auf die Forschungsfrage I.2 lässt sich zusammenfassend feststellen, dass in beiden hier untersuchten Probandengruppen (Erst- und Drittklässler) in jeweils beiden Sprachen signifikante positive Korrelationen zwischen der Anzahl der in den Narrativen produzierten Verben und SS bestehen, teils gilt dies auch für $G A O$. Es bleibt jedoch ungeklärt, ob möglicherweise die Gesamtlänge der Geschichte genauso entscheidend ist für die Ergebnisse, da keine signifikanten Korrelationen für genormte Werte, das heißt in Prozent, gefunden worden sind. Jedoch bilden sich im Vergleich zu den Nomen dennoch verbspezifische Tendenzen heraus. Besonders interessant sind diesbezüglich die Ergebnisse in der Gruppe der Drittklässler: Im Russischen gab es für Verben, aber nicht für Nomen, (Tokens und Lemmata) hohe Korrelationen mit SS. Auch bei den Erstklässlern bestehen bei Deutsch etwas stärker ausgeprägte positive Korrelationen mit den Verben. Des Weiteren betrifft die einzige positive Korrelation, die mit IST in Prozent gefunden wurde, die Anzahl der VerbLemmata (bei den Drittklässlern im Deutschen), während keine Korrelation mit den Nomen (weder Tokens noch Lemmata) besteht.

Zusammenfassend betrachtet decken sich die Ergebnisse des Teils I insofern mit dem, was die bisherige Forschung gezeigt hat, dass weniger und schwächere Korrelationen zwischen den lexikalischen Fähigkeiten und $S C$ gefunden wurden als mit der quantitativen Komponente $S S$, was für graduelle Unterschiede in der Sprachabhängigkeit spricht (vgl. Gagarina 2016). 
Die sprachübergreifende Untersuchung (Teil II) hat ergeben, dass die $S S$ zwischen den Sprachen positiv korreliert, obwohl die meisten Probanden beim Verbwortschatz in einer Sprache im Verhältnis zur Probandengruppe (Vorschüler) besser waren. Ähnliche Ergebnisse fand Pearson (2002) beim Vergleich zwischen Englisch und Spanisch: Korrelationen zwischen den Sprachen bei Narrativen (composite narrative score), aber nicht beim Sprachstand (language score). Daraus schließt Pearson (2002), dass bilinguale Kinder Kenntnisse von der Struktur einer Geschichte zwischen den Sprachen in höherem Maße übertragen können, als es bei anderen Domänen wie beim Lexikon der Fall ist.

Da Teil II.3 gezeigt hat, dass sich das Verhältnis zwischen dem Verbwortschatz beider Sprachen (Dominanz in einer Sprache vs. ausgeglichener Verbwortschatz) nicht in Unterschieden zwischen der SS in beiden Sprachen niederschlägt, kann nicht behauptet werden, dass sich allein anhand des Verbwortschatzes voraussagen lässt, in welcher Sprache bilinguale Probanden höhere Punktzahlen bei $S S$ erreichen.

Als Grenzen dieser Studie und damit der Belastbarkeit der Ergebnisse ist die Anzahl an Probanden pro Gruppe zu nennen sowie die Tatsache, dass die Wortschatztests, auf denen ein großer Teil der Analysen beruht, nur ein Annäherungsmaß des tatsächlichen gesamten produktiven Verbwortschatzes darstellen. Die Verben, die in den Wortschatztests vorkommen, sind möglicherweise nicht die für die Makrostruktur relevanten, sondern spielen eher auf stilistischer Ebene eine Rolle (abwechslungsreiche Wortwahl, präzisere Darstellung etc.). Für die Geschichte wesentliche „einfachere“ Verben wie z.B. essen waren vor allem bei den deutschen Sprachstandstests verhältnismäßig wenig vertreten. Die Ergebnisse dieser Studie sprechen dafür, dass für die Makrostruktur die allgemeinen kognitiven Fähigkeiten neben einem bestimmten Mindestmaß an produktivem Wortschatz ausschlaggebend sind. Die Geschichten von Kindern, die bereits über ein vielfältiges, ausdifferenziertes Verbrepertoire verfügen und beispielsweise jonglieren oder entgleisen im Wortschatztest produzieren konnten, sind vermutlich insgesamt zielsprachlicher. Jedoch können lexikalische Lücken durch Paraphrasierungen und weniger spezifische Verben umgangen werden. Dafür, dass trotz unterschiedlicher lexikalischer Fähigkeiten eine vergleichbare Makrostruktur erreicht werden kann, sprechen auch die Ergebnisse der Studie von Iluz-Cohen \& Walters (2012).

\section{Literaturverzeichnis}

Bishop, D. V. M., \& Edmundson, A. (1987). Language impaired 4-year-olds: Transient from persistent impairment. Journal of Speech and Hearing Disorders, 52, 156-173. 
Bretherton et al. (1981). Comprehension and production of symbols in infancy. Developmental Psychology, 17, 728-736.

Bruner, J. (1986). Actual Minds and possible Worlds. Cambridge, MA: Harvard University Press.

Clark, E. V. (2003). First language acquisition. Cambridge: CUP.

Elley,W. B. (1989). Vocabulary acquisition from listening to stories. Reading Research Quarterly, 24, 174-187.

Frawley,W. (1992). Linguistic semantics. Hillsdale, NJ: Lawrence Erlbaum Asssociates.

Gagarina, N. (2016). Narratives of Russian-German preschool and primary school bilinguals: rasskaz and Erzaehlung. Applied Psycholinguistics 37(1). 91-122.

Gagarina, N., Klop, D., Kunnari, S., Tantele, K., Välimaa, T., Balčiūnienė, I., Bohnacker, U., \& Walters, J. (2015). Assessment of Narrative Abilities in Bilingual Children. In Sharon Armon-Lotem, Jan de Jong \& Natalia Meir (Eds.), Assessing Multilingual Children. Bristol: Multilingual Matters, 243-269.

Gagarina, N., Klop, D., Kunnari, S., Tantele, K., Välimaa, T., Balčiūnienė, I., Bohnacker, U., \& Walters, J. (2012). MAIN - Multilingual Assessment Instrument for Narratives. ZAS Papers in Linguistics, 56.

Gagarina, N., Klassert, A., \& Topaj, N. (2010). Russian language proficiency test for multilingual children. ZAS Papers in Linguistics, 54.

Glück, C. W. (2007). Wortschatz- und Wortfindungstest für 6- bis 10-Jährige (WWT6-10). München: Elsevier.

Golinkoff, R. M., Hirsh-Pasek, K., Mervis, C. B., Frawley, W., \& Parillo, M. (1995). Lexical principles can be extended to the acquisition of verbs. In M. Tomasello \& W. Merriman (eds.), Beyond names for things: Young children's acquisition of verbs, 185-222. Hillsdale, NJ: Lawrence Erlbaum Associates.

Hayward, D., \& Schneider, P. (2000). Effectiveness of teaching story grammar knowledge to preschool children with language impairment. An exploratory study. Child Language Teaching and Therapy, 16(3), 255-284.

Heilmann, J., Miller, J. F., \& Nockerts, A. (2010). Sensitivity of narrative organization measures using narrative retells produced by young school-age children. Language Testing, 27, 603-626.

Hering, J. (2008). Vorlesen, Bilderbücher und die Entstehung von Erzählfähigkeit. Kind-BildBuch Zeitschrift des BIBF - Bremer Institut für Bilderbuch- und Erzählforschung (4), Universität Bremen.

Hockett, C. F. (1960). The Origin of Speech. Scientific American 203, 88-111.

Hughes, D., McGillvray, L., \& Schmidek, M. (1997). Guide to narrative language: Procedures for assessments. Eau Claire, WI: Thinking Publications.

Huttenlocher, J., Smiley, P. \&, Ratner, H. (1985). Was verraten Wortbedeutungen über die kognitive Entwicklung? In Thomas B. Seiler, \& Wolfgang Wannenmacher (Eds.), 
Begriffs- und Wortbedeutungsentwicklung. Theoretische, empirische und methodische Untersuchungen. Berlin: Springer, 211-230.

Iluz-Cohen, P., \& Walters, J. (2012). Telling stories in two languages: Narratives of bilingual preschool children with typical and impaired language. Bilingualism: Language and Cognition, 15(Special Issue 01), 58-74.

Kauschke, C. (2002). Entwicklung und Störungen des Verblexikons. In Forum Logopädie 2 (16), 6-10.

Kauschke, C. (2000). Der Erwerb des frühkindlichen Lexikons - eine empirische Studie $\quad z u r$ Entwicklung des Wortschatzes im Deutschen. Tübingen: Narr.

Kauschke, C. \& Siegmüller, J. (2010). Patholinguistische Diagnostik bei Sprachentwicklungsstörungen. München: Elsevier.

Komor, A. (2008). Semantische Basisqualifikation. In Konrad. C. Ehlich, Ursula. Bredel \& Hans. H. Reich (Eds.), Referenzrahmen zur altersspezifischen Sprachaneignung, 51-75. Bonn, Berlin: Bundesministerium für Bildung und Forschung.

Liles, B. Z. (1987). Episode organization and cohesive conjunctives in narratives of children with and without language disorder. Journal of Speech and Hearing Research, 30, 185-196.

Liles, B. Z. (1993). Narrative discourse in children with language disorders and children with normal language: A critical review of the literature. Journal of Speech and Hearing Research, 36, 868-882.

Liles, B. Z., Duffy, R. J., Merritt, D. D., \& Purcell, S. L. (1995). Measurement of narrative discourse ability in children with language disorders. Journal of Speech and Hearing Research, 38, 415-425.

MacWhinney, B. (2000). The CHILDES Project: Tools for analyzing talk. Mahwah, NJ: Lawrence Erlbaum Associates.

Mandler, G. (1979). Organization and repetition: Organization principles with special reference to rote learning. In L. Nilsson (ed.), Perspectives on memory research, 293-327. Hillsdale, NJ: Erlbaum.

McCabe, A. (1996). Evaluating discourse skills. In K. N. Cole, P. S. Dale, \& D. J. Thal (Eds.), Assessment of communication and language, 121-141. Baltimore, MD: Paul H. Brookes.

Pearson, B. Z. (2002). Narrative competence among monolingual and bilingual school children in Miami. In K. Oller \& R. Eilers (Eds.), Language and Literacy in Bilingual Children, 135-174. Clevedon, UK: Multilingual Matters.

Pearson, B. Z., \& S. Fernandez. (1994). Patterns of interaction in the lexical development in two languages of bilingual infants. Language Learning, 44, 617-653.

Pence, K, Golinkoff, R. M., Brand, R., \& Hirsh-Pasek, K. (2005). When actions can’t speak for themselves: How might infant-directed speech and infant-directed action influence verb learning? In T. Trabasso, J. Sabatini, D. W. Massaro \& R. C. Calfee (Eds.), From orthography to pedagogy: essays in honor of Richard L. Venezky, , 63-79. Mahwah, NJ: Lawrence Erlbaum Associates. 
Quasthoff, U. M. (1980). Erzählen in Gesprächen: Linguistische Untersuchungen zu Strukturen und Funktionen am Beispiel einer Kommunikationsform des Alltags. Tübingen: Narr.

R Core Team (2014). R: A language and environment for statistical computing. R Foundation for Statistical Computing, Vienna, Austria. URL http://www.R-project.org/.

Roth, F. (1986). Oral narrative abilities of learning-disabled students. Topics in Language Disorders: 7 (1), 21-30.

Schelten-Cornish, S. (2008). Förderung der kindlichen Erzählfähigkeit. Idstein: SchulzKirchner Verlag.

Shapiro, L. R. \& Hudson, J. A. (1991). Tell me a make-believe story: Coherence and cohesion in young children's picture-elicited narratives. Developmental Psychology, 27(6), 960-974.

Smith, C. A., \& Sachs, J. (1990). Cognition and the verb lexicon in early lexical development. Applied Psycholinguistics, 11, 409-424.

Stein, N. L., \& Glenn, C. G. (1979). An analysis of story comprehension in elementary school children. In R. O. Freedle (ed.), Discourse processing: Multidisciplinary perspectives. Norwood, NJ: Ablex.

Swanson, L., Fey, M., Mills, C., \& Hood, L. (2005). Use of narrative-based language intervention with children who have specific language impairment. American Journal of Speech-Language Pathology, 14, 131-143.

Westby, C. E. (2005). Assessing and facilitating text comprehension problems. In H. Catts \& A. Kamhi (Eds.), Language and reading disabilities. Boston, MA: Allyn \& Bacon.

Westby, C. (1991). Learning to talk - talking to learn: Oral-literate language differences. In C. S. Simon (Ed.), Communication skills and classroom success (pp. 181-218). San Diego, CA: College-Hill.

\section{Anhang}

\section{Beispiele für mit MAIN elizitierte Narrative}

Erklärung der in den Transkriptionen verwendeten Zeichen: [*] und @m kennzeichnen nichtzielsprachlich verwendete Wörter und morphologische Fehler; [//] steht für eine Umformulierung; (.) für eine Pause; runde Klammern bei einem Wort weisen darauf hin, dass ein Laut nicht ausgesprochen wurde; [ =! 2x] kennzeichnet eine Wiederholung; +// eine abgebrochene Äußerung; \& markiert eine gefüllte Pause oder ein phonologisches Fragment.

Für alle Beispiele ist die gleiche Bildergeschichte $(B B)$ gewählt worden, damit sie untereinander verglichen werden können. Da für die Erst- und Drittklässler auch die Token- und Lemma-Anzahl der in den Narrativen produzierten Verben 
untersucht wurden, ist unter dem 2. und 3. Beispiel jeweils noch eine nach Wortarten sortierte Übersicht über die verwendeten Wörter zu finden.

\section{Beispiel}

Diese Geschichte weist schon einige Teile einer Geschichte auf, enthält aber noch keine vollständigen $G A O$-Episoden. Alter des Kindes: 4;2

*CHI: die mama $+/$.

*CHI: \&aehm (...) die mama sagt die [*] voegel@m tschuess und sie fliegte@m (.) so weit weg .

*CHI: die voegeln@m konnten nicht fliegen .

${ }^{*}$ CHI: sie wollten sie mehr milch und xxx da wollte die katze (.) sie auffressen .

*CHI: und die katze wollte den ein [*] voegel@m nehmen .

*EX1: einen nur?

*CHI: ja .

*CHI: warum die mama hat zwei voegel .

*CHI: und die katze klettert in [*] den baum .

*CHI: und < der hund > [/] (..) der hund siehte@m die katze .

*CHI: er wollte sie (.) fressen .

*CHI: und dann \&aeh \&mh katze angst und so schneller rennte@m sie weg .

*CHI: und dann (..) hat der hund (..) gebissen in den schwanz die katze $+/$.

*EX1: oh hoho \&=lacht .

${ }^{*} \mathrm{CHI}:+,<$ und der \&gro $>[/ /]$ und die grosse mama von denen hat angst .

\section{Beispiel}

Die Geschichte weist eine gute $S S$ und $S C$ auf, enthält aber verhältnismäßig wenige IST. Alter des Kindes: 7;11.

*CHI: es war einmal ein vogel und es [*] hatte zwei kleine vogelchen@m .

${ }^{*} \mathrm{CHI}$ : und einma(l) flog sie weg nach den $\left[{ }^{*}\right]$ essen und die zwei vogelchen $[*]$ blieben allein .

*CHI: dann kam ein hungriger kater und er \&kle $+/ /$.

*CHI: und wann [*] ihre mutter schon da war kletterte er auf dem [*] baum .

*CHI: also die mutter war mit einem wurm da und der kater kletterte auf den baum .

${ }^{*} \mathrm{CHI}$ : und dann wann [*] sie den [*]< anderen $\& \mathrm{kl}>[/ /]$ einen kleinen vogelchen@m essen gegeben hat dann war der kater ganz oben und hat $+/ /$.

${ }^{*} \mathrm{CHI}$ : er wollte einen kleinen vogel also mitnehmen nach unten und dann auf friss@m.

*CHI: dann da sah es der hund .

*CHI: hm < er ist $>$ [//] und dann hat er ihn an [*] den schwanz gebissen und ihn $\mathrm{xxx}$. 
${ }^{*} \mathrm{CHI}$ : und die $[=! 2 \mathrm{x}]$ mutter von den kleinen voegelchen die hat sie beschuetzt ein bisschen damit wenn er wieder hochklettert .

${ }^{*} \mathrm{CHI}$ : und wann [*] der hund ihn weggejagt hat von dem baum war(e)n die zwei voegelchen und ihre mutter sehr froehlich .

Übersicht über die in dieser Geschichte verwendeten Wörter (sortiert nach Wortarten):
1 ADJ|ander-en
4 DET:art:def|d-ie
5 PRO|er
1 ADJ|hungrig-er
3 DET:art:indefeein
3 PRO|er-ACC
1 ADJ|klein-e
1 DET:art:indeflein-em
3 PRO|es
3 ADJ|klein-en
1 DET:art:indef|ein-en
2 PRO|sie
3 ADV:wh|wann
2 DET:pro:poss|ihr-e
1 PRO|sie-PL
1 ADV|allein
$2 \mathrm{~N}: 03: \mathrm{m} \mid$ hund
$1 \mathrm{~V}: 01 \mid$ beschuetz-PP
2 ADV|also
$3 \mathrm{~N}: 04: m \mid$ baum
3 ADV|da
$1 \mathrm{~N}: 04: \mathrm{m} \mid \mathrm{sch} w a n z$
2 V:01|kletter-PRET:3S
$6 \mathrm{ADV} \mid \mathrm{dann}$
2 ADV|einmal
1 ADV|froehlich
1 ADV|ganz
1 ADV|oben
$1 \mathrm{ADV} \mid$ schon
$3 \mathrm{~N}: 05: \mathrm{m} \mid$ kater
$1 \mathrm{~V}: 03 \mid$ beiss-PP
$2 \mathrm{~N}: 05: \mathrm{n} \mid$ essen
$1 \mathrm{~V}$ :04|bleib-PRET:3P
$2 \mathrm{~N}: 05: n \mid$ vogel\&DIM3-
$1 \mathrm{~V}: 10 \mid$ geb-PP
1 ADV|sehr
1 ADV|unten
$1 \mathrm{ADV} \mid$ wieder
PL
$1 \mathrm{~V}: 10 \mid$ seh-PRET:3S
$3 \mathrm{~N}: 05: \mathrm{n} \mid$ vogel\&DIM3-
$1 \mathrm{~V}: \mathrm{S} \mid \mathrm{hab}-3 \mathrm{~S}$
$1 \mathrm{~V}: S \mid$ hab-PRET:3S
PL*m
$1 \mathrm{~V}: S \mid$ sein-PRET:3P
$4 \mathrm{~N}: 06:$ f|mutter
$4 \mathrm{~V}: S \mid$ sein-PRET:3S
$2 \mathrm{~N}: 06: \mathrm{m} \mid$ vogel
$1 \mathrm{~N}: 08: m \mid$ wurm
$1 \mathrm{~V}: X \mid k o m m-P R E T: 3 S$
1 CO:assjja
3 NUM|zwei
4 V:aux|hab-3S
$1 \mathrm{CONJ}$ /damit
1 PREP|an
$1 \mathrm{~V}: \bmod \mid$ woll-PRET:3S
$14 \mathrm{CONJ} /$ und
2 PREP|auf
$1 \mathrm{CONJ}$ |wenn
1 PREP|mit
$1 \mathrm{CO} \mid \mathrm{hm}$
2 PREP|nach
2 DET:art:def|d-em
2 PREP|von
1 hoch\#V:01|kletter-3S
1 mit\#V:11|nehm-INF
1 weg\#V:01 jag-PP
1 weg\#V:06|flieg-
PRET:3S
5 DET:art:def|d-en
1 PRO:qn|bisschen
4 DET:art:def|d-er
1 PRO:rel|d-ie

\section{Beispiel}

Dieses Beispiel zeigt eine Geschichte, die hinsichtlich aller drei untersuchten Makrostrukturkomponenten eine weit entwickelte narrative Fähigkeit widerspiegelt. Alter des Kindes: 7;0

*CHI: eine vogelmama hatte $[=! 2 \mathrm{x}]$ schon laengst eier bekommen und kueken bekommen .

*CHI: die waren hungrig . 
*CHI: die mama flog weg um [=! 2x] ihre babys zu fuettern .

*CHI: aber sie bemerkte es nicht dass unten sich eine gefahr vorstellte .

*CHI: denn eine katze sah die \&k kleinen armen kuekens@m und wollte sie auffressen .

*CHI: die mama kam mit einen [*] leckeren wurm zurueck und gab sie den an und bemerkte nicht dass die katze hochkletterte .

*CHI: sie [=! 2x] fuetterte das [=! 2x] baby das ganz hinten war .

*CHI: aber dafuer bemerkte sie nicht dass das baby das ganz vorne war von der katze fast runtergerissen wurde .

*CHI: aber dafuer $+/ /$.

${ }^{*} \mathrm{CHI}$ : zum glueck kam noch ein hund und hat es gesehen und riss die katze $<$ mit ihrem $>[/ /]$ mit $\left[{ }^{*}\right]$ dem schwanz hinunter .

*CHI:er jagte die katze ganz weit weg .

${ }^{*} \mathrm{CHI}$ : und die mutter war wieder zufrieden mit ihren kindern .

Übersicht über die in dieser Geschichte verwendeten Wörter, sortiert nach Wortarten:
1 ADJ|arm-en
2 DET:art:def|d-as
$1 \mathrm{~N}: 07: \mathrm{n} \mid$ kind-PL-DAT
$1 \mathrm{ADJ} /$ hungrig
2 DET:art:def|d-em
$1 \mathrm{~N}: 08: \mathrm{m} \mid$ wurm
$1 \mathrm{ADJ} /$ klein-en
1 DET:art:def|d-er
3 PREP|mit
1 ADJ|lecker-en
7 DET:art:def|d-ie
1 PREP|von
2 ADV:pro|dafuer
1 DET:art:indef|ein
1 PREP|zu
1 ADV|aber
1 ADV|denn
3 DET:art:indef|ein-e
1 PRO:dem|d-en
1 DET:art:indef|ein-en
1 PRO:dem|d-ie
1 ADV|fast
3 ADV|ganz
1 DET:pro:poss|ihr-e
1 PRO:refl|man-ACC
1 DET:pro:poss|ihr-en
2 PRO:rel|d-as
1 ADV|hinten
$1 \mathrm{INF} \mid \mathrm{zu}$
1 PRO|er
1 ADV|laengst
3 ADV|nicht
$2 \mathrm{~N}: 01: f \mid m a m a$
2 PRO|es
$1 \mathrm{~N}: 01: f \mid$ vogel+mama
4 PRO|sie
1 ADV|noch
1 ADV|schon
$2 \mathrm{~N}: 01: n \mid$ baby
$1 \mathrm{PRO} \mid$ sie-PL
1 ADV|unten
$1 \mathrm{~N}: 01: n \mid$ baby-PL
1 PTL|an
1 ADV|vorne
$1 \mathrm{~N}: 02: f \mid g e f a h r$
$3 \mathrm{~V}: 01 \mid$ bemerk-PRET:3S
$5 \mathrm{~N}: 02: \mathrm{f} \mid \mathrm{katze}$
$1 \mathrm{~V}: 01 \mid$ fuetter-INF
1 ADV|weit
$1 \mathrm{~N}: 03: \mathrm{m} \mid$ hund
$1 \mathrm{~V}: 01 \mid$ fuetter-PRET:3S
$1 \mathrm{ADV} \mid$ wieder
1 ADV|zufrieden
$1 \mathrm{~N}: 03:$ :nglueck
$1 \mathrm{~V}: 10 \mid$ geb-PRET:3S
$1 \mathrm{~N}: 04: \mathrm{m} \mid$ schwanz
$1 \mathrm{~V}: 10 \mid$ seh-PP
2 CONJ|aber
$1 \mathrm{~N}: 05: n \mid k u e k e n-P L$
$1 \mathrm{~V}: 10 \mid$ seh-PRET:3S
$3 \mathrm{CONJ} /$ dass
$1 \mathrm{~N}: 05: n \mid k u e k e n-P L 1 * m$
$1 \mathrm{~V}: S \mid$ sein-PRET:3P
$1 \mathrm{CONJ} \mid \mathrm{um}$
$1 \mathrm{~N}: 06: f \mid m u t t e r$
$3 \mathrm{~V}: \mathrm{S} \mid$ sein-PRET:3S
$7 \mathrm{CONJ} /$ und
$1 \mathrm{~N}: 07: n \mid e i-P L$
$2 \mathrm{~V}: \mathrm{X} \mid$ bekomm-PP 
$1 \mathrm{~V}: X \mid$ komm-PRET:3S

$1 \mathrm{~V}$ :aux |hab-3S

$1 \mathrm{~V}$ :aux|hab-PRET:3S

$1 \mathrm{~V}$ :aux|werd-PRET:3S

$1 \mathrm{~V}$ :mod|woll-PRET:3S

1 auf\#V:10|fress-INF

1 hinunter\#V:03|reiss-

PRET:3S

1 hoch\#V:01|kletter-

PRET:3S

1 runter\#V:03|reiss-PP

1 vor\#V:01|stell-

PRET:3S

1 weg\#V:01 jag-

PRET:3S

1 weg\#V:06|flieg-

PRET:3S

1 zurueck\#V:X|komm-

PRET:3S 